\title{
Five cases of isolated glycerol kinase deficiency, including two families: failure to find genotype:phenotype correlation
}

\author{
Carole A Sargent, Alexa Kidd, Sue Moore, John Dean, Guy T N Besley, Nabeel A Affara
}

\begin{abstract}
Little is understood of the genotypel phenotype correlations in $\mathrm{X}$ linked glycerol kinase deficiency (GKD) where most cases are caused by extensive deletions of Xp21, which often include genes flanking the $G K$ locus. Few cases of isolated GKD have been investigated where the phenotype is not influenced by neighbouring genes. In this paper, we present the mutation data from four confirmed and one suspected case of non-deletion, isolated, $X$ linked GKD and therefore extend the base of patients that can allow an assessment of genotype/phenotype correlations for this disease. The mutations found were two terminations leading to premature truncation of the GK polypeptide chain, one insertion, and an amino acid substitution. Phenotypic variation was observed in two families, where there was more than one affected subject carrying the same mutation, confirming previous studies that suggest there is no correlation between disease severity and genotype. Furthermore, the nature of the mutation in different families does not appear to influence the spectrum of phenotypic variation. In addition, one coding polymorphism in exon 3 has been found. The characterisation of the gene structure has been completed and shows that instead of 19 there are 21 exons.

(f Med Genet 2000;37:434-441)
\end{abstract}

Keywords: glycerol kinase deficiency; genotype/ phenotype correlation

Glycerol kinase deficiency $(\mathrm{GKD})^{1}$ is an $\mathrm{X}$ linked disorder resulting from the deletion or mutation of the gene for the enzyme glycerol kinase (E.C.2.7.1.30). Normally, the enzyme catalyses the phosphorylation of dietary glycerol to glycerol-3-phosphate, which is used in the synthesis of lipids. At the mitochondrial outer membrane, competition between glycerol kinase and hexokinase is believed to be important for the regulation of the balance between carbohydrate and lipid metabolism. Loss of enzyme activity is characterised by a raised level of free glycerol in both the urine (hyperglyceroluria) and the serum (hyperglycerolaemia) of an affected person. The severity of the disease is highly variable; the severe infantile form results from a contiguous gene deletion syndrome involving loss of the gene for glycerol kinase (GK) with all or part of the genes for Duchenne muscular dystrophy
(DMD) or congenital adrenal hypoplasia (AHC) or both. In contrast, isolated GKD is not associated with AHC or DMD, and can manifest as an adult, benign ${ }^{2}$ form or a juvenile form. The juvenile form shows phenotypic variation both between subjects from different families and within families. ${ }^{3-5}$ In some cases it can be similar in the degree of severity to the infantile form. This has led to the proposal that genetic background and environmental factors have important modifying influences. ${ }^{45}$

The gene for GKD is one of a family that includes intronless testis specific autosomal copies. $^{6-9}$ The $\mathrm{X}$ linked gene is the only member of the family to have introns. In total, there are 21 exons, two of which are subject to alternative splicing, and a common transcript size of $2 \mathrm{~kb}$ in somatic tissues.

In this study we have looked at the cause of isolated GKD in four confirmed non-deletion families and a single suspected case. All patients and families were referred for biochemical analysis following episodes of hypoglycaemic coma, with seizures also occurring in two subjects. On confirmation of the diagnosis, molecular techniques were used to define the types of mutation present. RT-PCR was used to sequence the whole gene product of two patients. The remaining cases were investigated using a combination of exon specific amplification, SSCP analysis, and sequencing. The data have been combined with a survey of other published cases to look at the distribution of mutations across the gene structure. This confirms that each represents a novel, family specific mutation. One polymorphism in exon 3 has also been defined.

\section{Materials and methods}

PATIENTS

Patients 1 and 2 are non-identical twin boys. One brother showed no apparent symptoms, whereas the second was diagnosed after several seizures. Patients 3 and 4 were both referred for biochemical analysis after suffering episodes of hypoglycaemic coma following periods of illness/fasting. These four cases were biochemically confirmed with glycerol kinase deficiency in the absence of raised creatine kinase activity or adrenal malfunction. These results suggested the patients had isolated glycerol kinase deficiency with no large associated deletions of the X chromosome. Patient 4 had reduced levels of GK enzyme activity in skin cell cultures. Southern blot hybridisation experiments, previously described for cases 1, 2, and $3,{ }^{6}$ established that the GK gene was not deleted 
or altered in the DNA from any of these patients. Cell lines were established for all four cases.

Patient 5 presented at 2 years of age, when he was found in a hypoglycaemic coma after a 17 hour fast. There was no significant past history apart from a tendency to be sweaty and irritable before breakfast. He responded rapidly to intravenous glucose. During the coma his blood sugar was $1.1 \mathrm{mmol} / 1$, serum bicarbonate was low (13 mmol/l), urinary organic acid screening showed very high glycerol levels, and serum triglyceride levels were very high (8.8 $\mathrm{mmol} / \mathrm{l})$. Glycerol kinase deficiency was later confirmed by finding very high levels of plasma glycerol $(4.6 \mathrm{mmol} / \mathrm{l})$ and a glycerol kinase enzyme activity of only $12 \%$ in skin fibroblasts. Complex glycerol kinase deficiency was ruled out by finding normal creatine kinase levels, normal adrenal function, and normal karyotype. A cell line was established for this patient. Nine other family members were subsequently assessed. None of them had any symptoms suggestive of GK deficiency but the proband's maternal grandfather (II.1) and great uncle (II.3) had very high plasma triglycerides and plasma and urinary glycerol levels. They also had raised cholesterol levels, and II. 1 has since had a myocardial infarct. Four other males at $50 \%$ risk had no biochemical evidence of GKD (II.5, IV.1, IV.3, IV.4). Three obligate carriers had undetectable plasma glycerol levels and urinary glycerol levels just above the upper limit of normal. DNA from all nine family members was made available for investigation.

Patient 6 was the offspring of a consanguineous marriage, suffered neonatal seizures, and died shortly after birth. Raised urinary glycerol was suspected and DNA was sent for further investigation of the glycerol kinase gene. No cell line was established for this patient.

SCREENING THE PAC LIBRARY FOR GENOMIC CLONES

The RPCI1 library was screened by PCR using primers for glycerol kinase exon 7 . This exon was previously determined to lie approximately in the middle of the genomic structure and was not represented in the previously isolated cosmid clones. Two PAC clones were isolated by this method, $7 \mathrm{e} 22$ and 2117 . DNA was prepared from the PAC clones using the standard protocol supplied by the HGMP resource centre. An additional phenol:chloroform extrac- tion and ethanol precipitation was used to remove RNase before the DNA was subjected to restriction digestion and PCR. Both clones were found to contain full length copies of the $X p$ gene, as shown by PCR of each exon from the isolated insert DNA.

PCR AMPLIFICATION OF EXONS

Primer pairs for exon amplification were as previously described ${ }^{7}$ except for exon 1 (primers GK33, ctc gtc acc tgc ccc tcc c, with GK26B, aca gcc acc ccc tcc gtc ccc cg), exon 8A (primers GKE8AA, aat agt ttc aca gga tgg tta g, with GKE8AB, aaa gcc caa agc aca tca cag tt), exon 9 (primers GKE9A, tgc ttt ctt tgt ctt gtc tgc, with GKE9B, gca cac tga tga act aag agg), exon 9A (primers GKE9AA, aaa atc cta tgg ctc ttc taa, with GKE9C, ttc aaa gca cca agt aga aaa a), exon 10 (primers GKE10A, ttg tct tac tat tca ttc tcc ct, with GKE10B, tta aac cct gat aaa gat taa $\mathrm{g}$ ), exon 11 (primers GKE11A, atc ttt tca ttt tcc act act g, with $\mathrm{GKE} 11 \mathrm{~B}^{7}$ ), exon 17 (primers GKE17A, ${ }^{7}$ with GKE17C, tgt gac aat aat aaa caa aaa aac), and exon 19 (primers GKE19A2, tgc ttt aac tcc tga att gta, with GK19R, gat gtt atg gca atg gag ggg). PCR reactions were carried out in the presence of $250 \mathrm{nmol} / 1$ primers, $200 \mu \mathrm{mol} / 1 \mathrm{dNTPs}$, and 1 $\times$ buffer $(10 \mathrm{mmol} / \mathrm{l}$ Tris-HCl, $\mathrm{pH} 9.0,1.5$ $\mathrm{mmol} / 1 \mathrm{MgCl}_{2}, 50 \mathrm{mmol} / 1 \mathrm{KCl}, 1 \%$ Triton $\mathrm{X}-100$, and $0.1 \%$ gelatin) with 0.5 units of "Super Taq" Taq polymerase (HT Biotechnology Ltd, Cambridge, UK). Each $50 \mu 1$ reaction contained $100 \mathrm{ng}$ of total human DNA. Cycling conditions for all PCRs were $95^{\circ} \mathrm{C}$ for 2.5 minutes followed by 35 cycles of $95^{\circ} \mathrm{C}$ for 20 seconds, $\mathrm{T}_{\text {anneal }}$ for 30 seconds, $72^{\circ} \mathrm{C}$ for 30 seconds, and a final extension at $72^{\circ} \mathrm{C}$ for 10 minutes. The $\mathrm{T}_{\text {anneal }}$ used for exon 19 primers was $57^{\circ} \mathrm{C}$, and for all other primer pairs it was $55^{\circ} \mathrm{C}$.

The sequence from intron 3 was amplified using primers ISF (atg tgt tgg ctt tct gga cc) and ISR (aag caa aag ctt ggt cct tg). PCR reactions were carried out as above, using an annealing temperature of $58^{\circ} \mathrm{C}$.

PCR products were purified before SSCP or sequence analysis using the high pure PCR product purification kit from Boehringer Mannheim $\mathrm{GmbH}$.

RT-PCR

RNA was prepared from cultured fibroblast lines $\left(10^{6}\right.$ to $10^{7}$ cells) using Tri-reagent (Sigma).

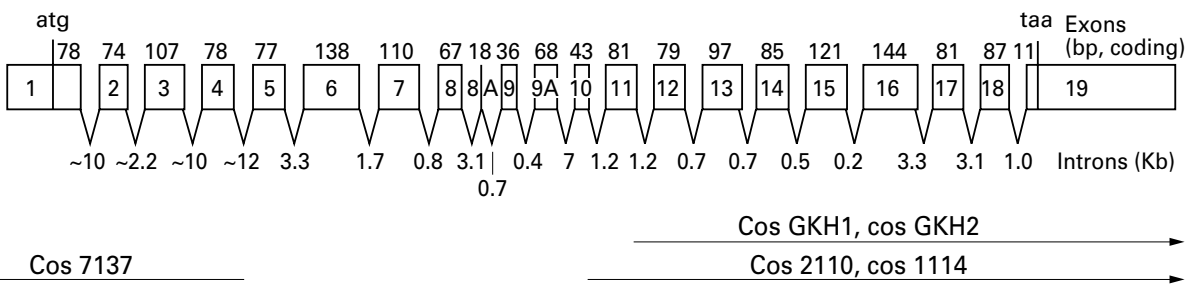

PAC 7e22, PAC 2I17, YAC 628F5

Figure 1 Schematic of the Xp glycerol kinase gene structure. Exons are represented by the numbered boxes and intron positions by the Vs. The numbers of bases coding are shown above the exons. Exon 1 has 114 bases of 5'UTR before the initiating atg. The exact size of exon 19 is undetermined, as there are three potential polyadenylation signals. Two of the three signals have been sequenced and are included in the annotation to GenBank entry X78211. The intron sizes are given below in kb, based on sizes of PCR products between exons. Genomic clones (cosmids, PACs, and a YAC) used to determine the gene structure are delineated beneath the schematic. ${ }^{7}$ 
The RNA was treated with Rnase free DNase before first strand cDNA synthesis using the Promega Reverse Transcription System. The cDNA was used in subsequent PCR reactions with intronic primers (GK exon 7) and GAPDH primers to test for DNA contamination and for cDNA quality respectively. The 5' portion of the $G K$ transcript was amplified using primers GK33 with GK30, and the 3' portion was amplified using primers GK29 with GK15. ${ }^{7}$
PCR reactions were carried out using $1 \mu \mathrm{l}$ of RT product in $20 \mu \mathrm{l}$ for 35 to 40 cycles at an annealing temperature of $55^{\circ} \mathrm{C}$.

SSCP ANALYSIS OF EXONS

Aliquots $(3 \mu \mathrm{l})$ of PCR product for each exon were denatured in formamide loading buffer (95\% formamide, $0.05 \%$ bromophenol blue, $0.05 \%$ xylene cyanol), snap cooled on ice, then run on $8 \%$ acrylamide, $10 \%$ glycerol, $0.5 \times \mathrm{TBE}$

Patient 5

A

N

C T A A A C A C

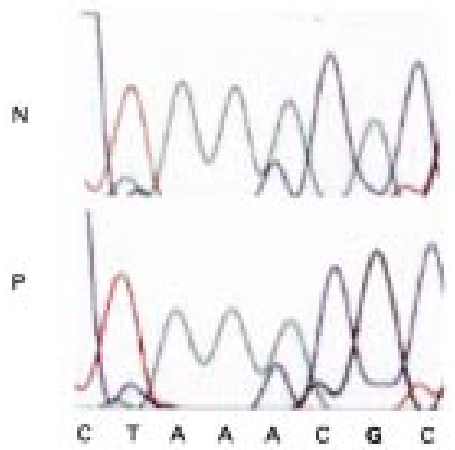

B

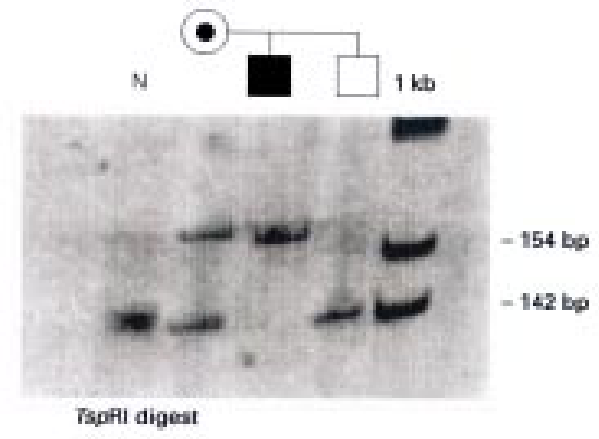

Fppll digest

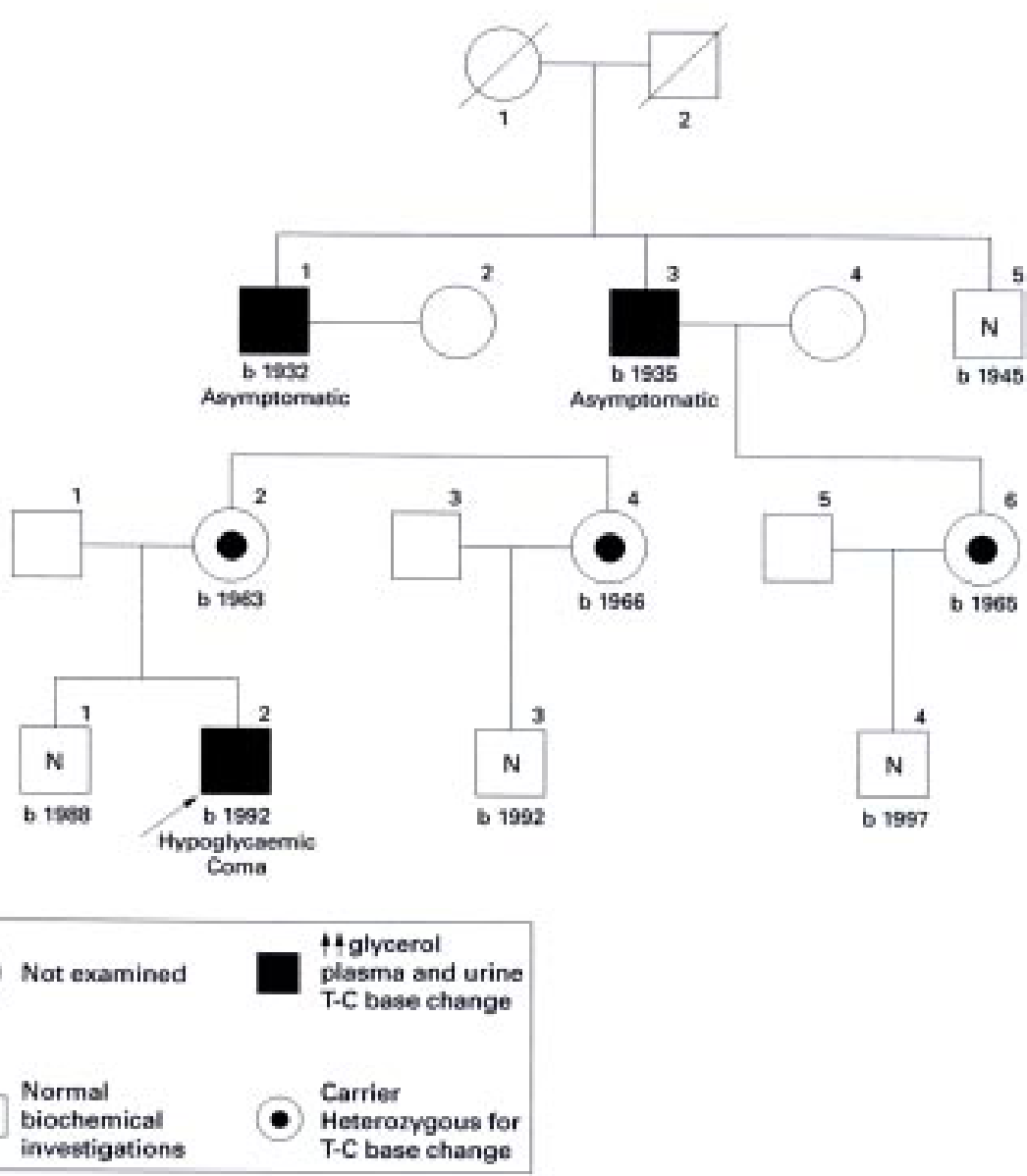

II

C I

III

N

Figure 2 Analysis of patient 5 and his extended family. $(A)$ Sequence difference between patient 5 ( $P$ ) and a normal control $(N)$, on the complementary strand of exon $9 A$. (B) TspRI digest of PCR product for exon $9 A$, confirming the inheritance of the normal (lower) and abnormal (upper) bands in two male sibs from a heterozygous mother. An unrelated subject is shown (N) and the marker is a $1 \mathrm{~kb}$ ladder. (C) Extended pedigree. Living subjects shown have been tested at the DNA level and biochemically, as indicated in the key. 
on $8 \times 10 \mathrm{~cm}$ minigels. Gels were run at $4^{\circ} \mathrm{C}$ in $0.5 \times \mathrm{TBE}$ at $1 \mathrm{~W}$ for three to four hours. Bands were visualised by silver staining. ${ }^{10}$

DNA SEQUENCING

Exons were sequenced with radioactive ${ }^{33} \mathrm{P}$ using the Amersham Thermo Sequenase radiolabelled terminator cycle sequencing kit and run on standard denaturing gels in glycerol tolerant buffer supplied by the manufacturer, or by fluorescent labelling using the Amersham Pharmacia Thermo Sequenase dye terminator cycle sequencing kit. Fluorescent sequences were separated on an ABI 373A DNA sequencer.

\section{Results}

REFINEMENT OF THE GENE STRUCTURE

Previous analysis of the glycerol kinase deficiency gene on the $\mathrm{X}$ chromosome has shown that it consists of at least 19 exons. ${ }^{7}$ Isolation of genomic clones (PAC 7-e-22 and PAC 2-1-17) containing the entire gene from the RPCI1 PAC library ${ }^{11}$ has permitted the completion of the structure. This has shown that exon 9 is in fact two separate exons of $36 \mathrm{bp}$ and $68 \mathrm{bp}$ with an intron between nucleotides 879 and 880 (EMBL entry X78211) (as also found by Sjarif et $a \bar{P}$ ) and that the originally published boundary between exons 10 and 11 was incorrectly assigned owing to the presence of a coligation within the $5^{\prime}$ portion of exon 11 in cosmid clone 2110. The correct boundary has been established between nucleotides 990 and 991 . Exon 10 is thus $43 \mathrm{bp}$ and exon 11 is $81 \mathrm{bp}$. An additional $18 \mathrm{bp}$ exon described in a fetal brain transcript, ${ }^{67}$ which lies between exons 8 and 9 , and could not be mapped in an Xp derived YAC clone, ${ }^{7}$ has subsequently been observed as a genuine exon in RT-PCR products from patient cell lines (study). ${ }^{4}$ Furthermore, an equivalent $18 \mathrm{bp}$ exon has been observed in transcripts from fetal mouse brain. ${ }^{12}$ The position and sequence of this exon was confirmed from the PAC genomic clones. Thus the total number of exons is 21 . The gene structure is shown schematically in fig 1 .

ANALYSIS OF PATIENTS WITH ISOLATED GKD

Cell lines were available for five subjects (patients 1, 2, 3, 4, and 5) from four independent families, all with confirmed GKD. DNA only was obtained for a sixth patient with suspected GKD. Patients 1, 2, and 3 have been described previously, and are known to show no major deletions or rearrangements of the GKD locus. ${ }^{6}$ The remaining patients were referred as isolated cases of GKD following biochemical assessment of enzyme activity.

Initially RNA was prepared from all the cell lines, to amplify the GK transcript by RT-PCR. Samples from patients 4 and 5 only gave prod-

Patient 4

A
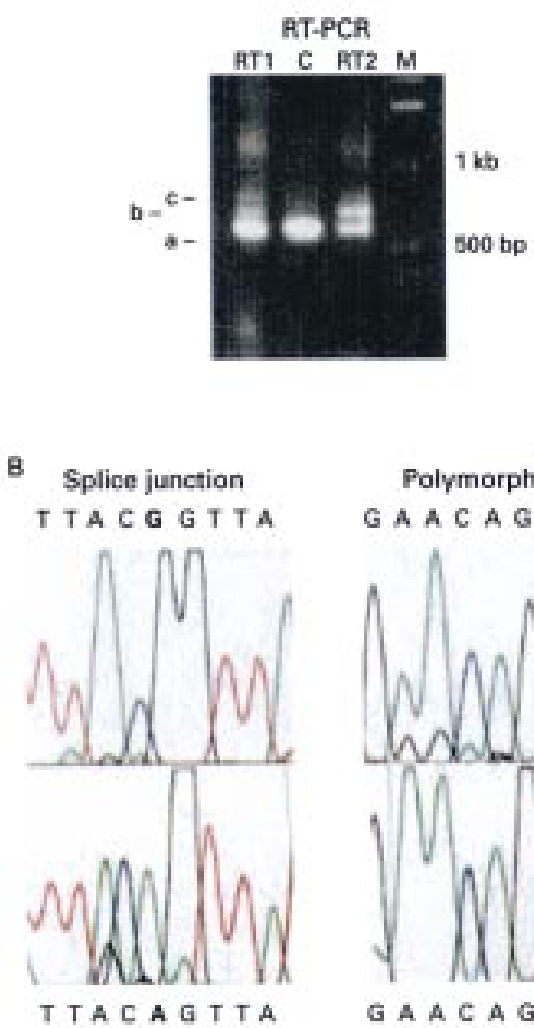

Polymorphism
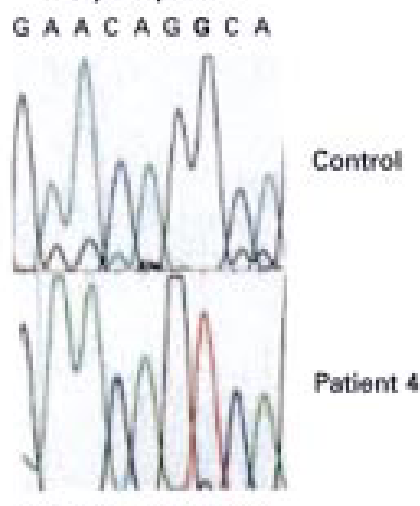

C

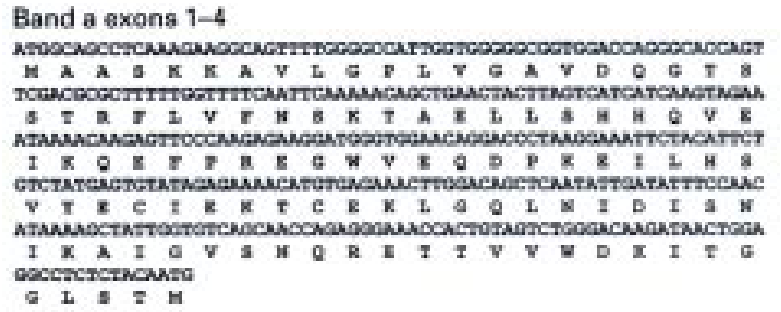

Band b exons 1,2, ins, 4 NTEDCADCCTCAM

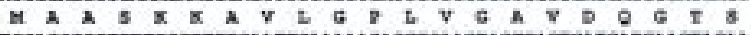

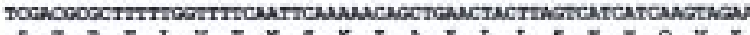

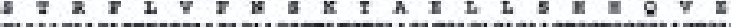

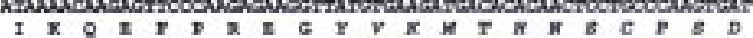

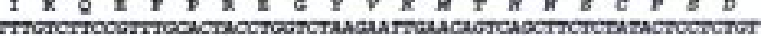
I S E V C T T Y S K N.

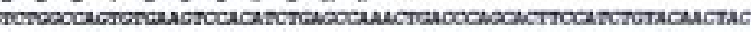

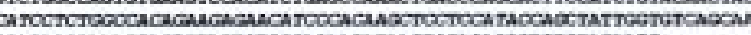

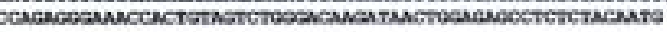

Band o exons 1,2,3, ins, 4

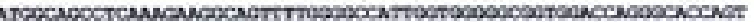
$M$ A A B K K A V L G V i V a A V D $Q$

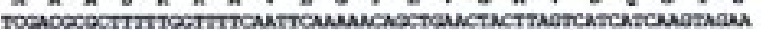

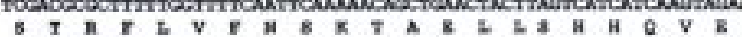

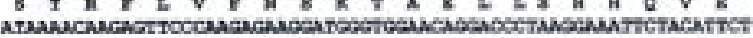

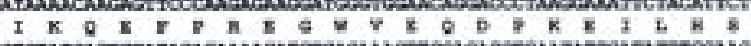

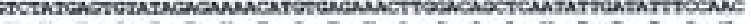

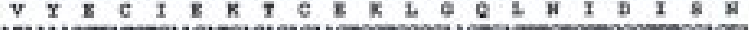

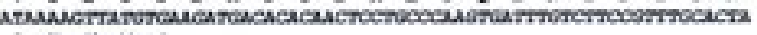

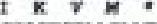

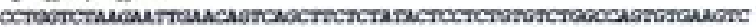

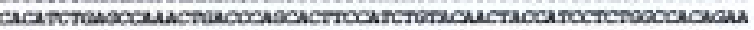

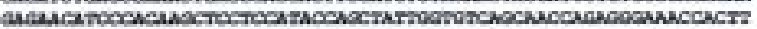

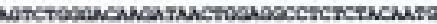

Figure 3 Analysis of patient 4 by RT-PCR and sequencing. (A) Results of two RT-PCR experiments (lanes RT1 and RT2), with the three bands from the patient $(a, b, c)$ compared with the single band in the normal control (lane $C$ ). (B) Sequence of the genomic fragment from intron 3 shows the creation of the splice junction on the left, and an internal polymorphism specific to the patient RT-PCR product is on the right, confirming the origin of the genomic PCR fragment. (C) Protein translations for the RT-PCR products, with early termination for the abnormal transcripts. The insertion is shown in italics. 
ucts. There appears to be a genuine deficit of the GK transcript in the other patient samples, as RT-PCR controls with GK primers using normal subjects, or controls on the patient cDNA with primers for other expressed genes give the expected PCR products.

Sequencing of the cDNA PCR product from patient 5 showed a $\mathrm{T}$ to $\mathrm{C}$ base substitution at the first nucleotide of exon 9A (nucleotide position 880 on EMBL entry X78211) that results in C256R (GTG to GCG) (fig 2A). This was confirmed by genomic PCR and sequence analysis. The base change also destroys a $T s p$ RI site in the genomic DNA (fig

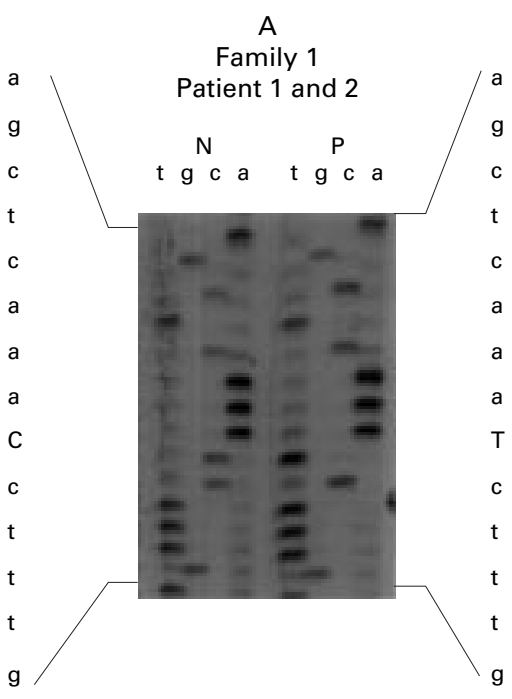

2B), offering an independent mode of assessment for this mutation. The RFLP was not seen in a screen of 40 other $\mathrm{X}$ chromosomes from normal subjects. Three generations of the proband's family were tested by both methods. The base change was found in the two other males who had the benign form of the condition; the grandfather (II.1) and the great uncle (II.3), but not in the males at a $50 \%$ prior risk, who all had normal glycerol levels. The three female obligate carriers were heterozygous for the mutation, as expected (fig 2C). ${ }^{13}$

As in a previous study of patients with isolated $\mathrm{GKD},{ }^{4}$ the $\mathrm{cDNA}$ prepared from

B

Patient 3

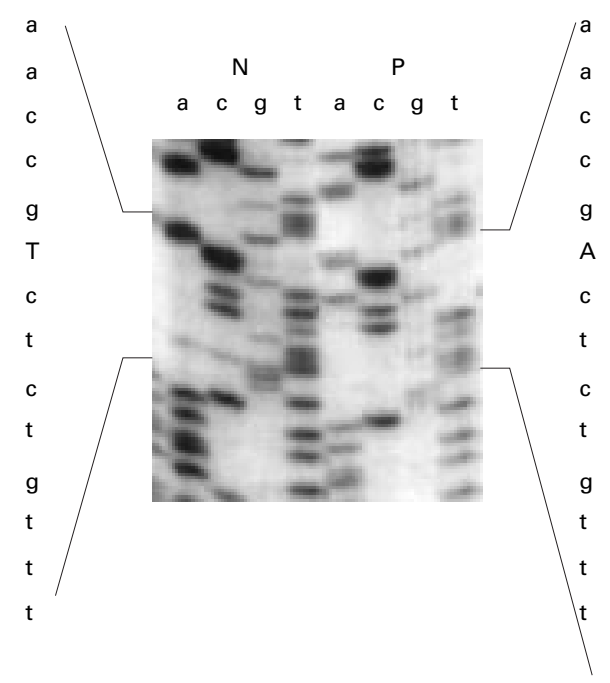

C

D

Family 3

Patient 6

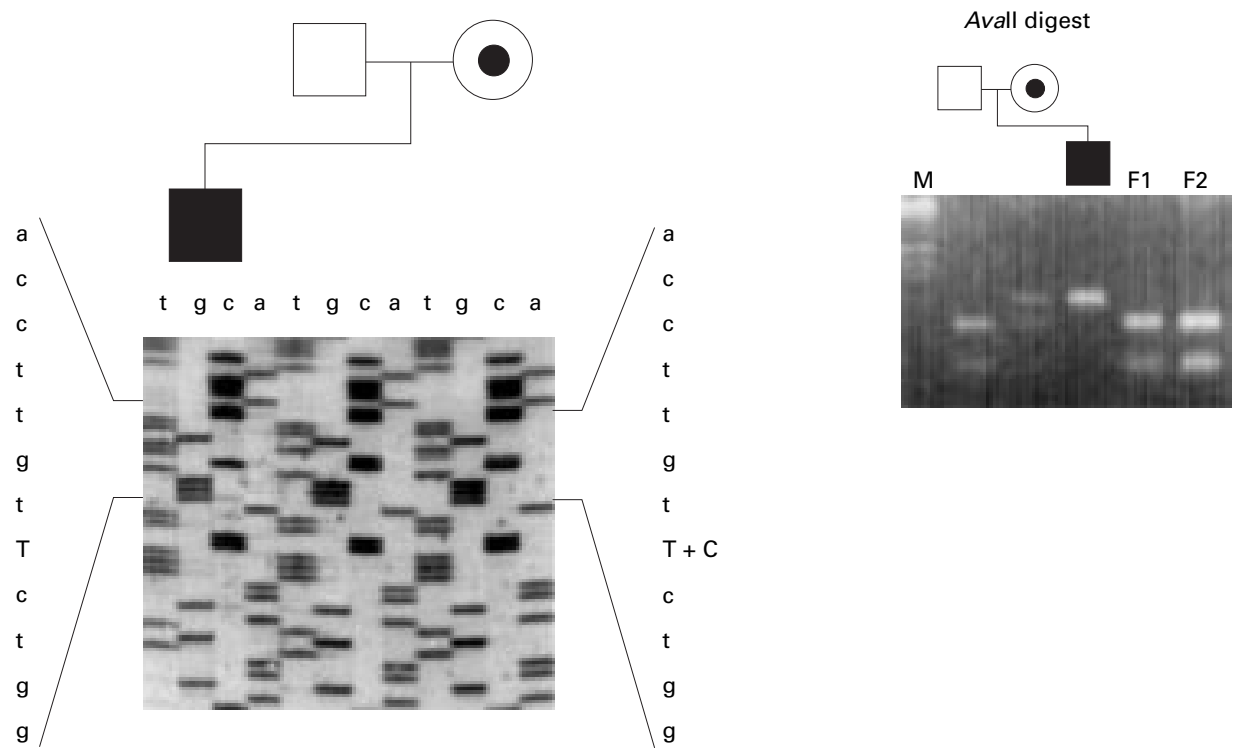

Figure 4 (A) Mutation analysis in patients 1 and 2. The sequence of the positive strand of exon 14 shows the codon change from CAA in the normal control $(N)$ to TAA in the patient (P) (only one brother is shown). (B) Patient $3(P)$ is compared with a control $(N)$ on the complementary strand for exon 14. The $T$ to $A$ highlighted represents a change in the codon from AGA to TGA. (C) Results for the family of patient 6 sequenced on the complementary strand of exon 3. His mother is heterozygous for the base at position $279(T+C)$, his father has the same sequence as that previously published (C), and patient 6 has the T. (D) The presence of the alternative codon is confirmed by digestion with AvaII. The marker (M) is a $1 \mathrm{~kb}$ ladder. Additional lanes show two unrelated female controls (F1 and F2). 


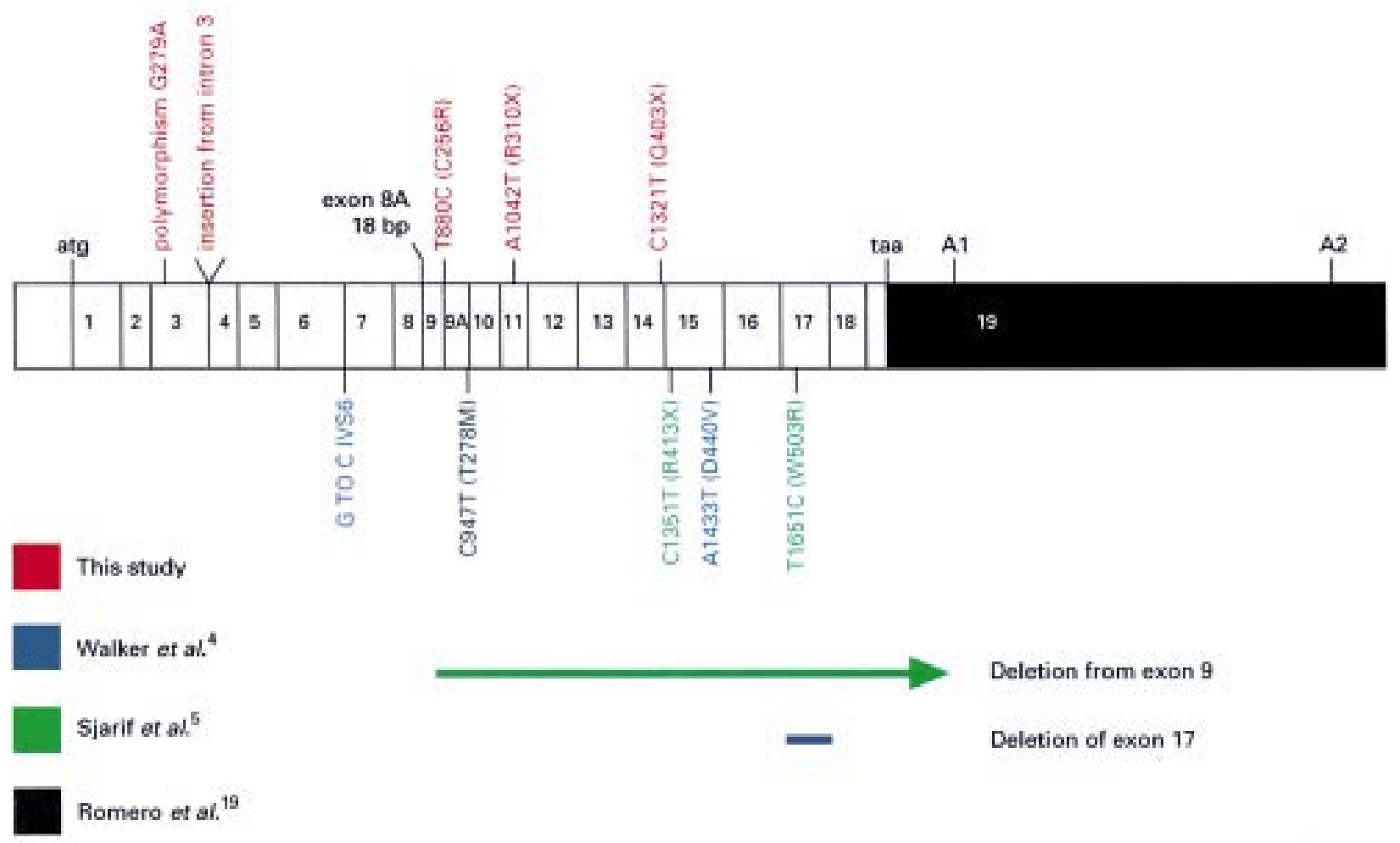

Figure 5 A summary of the glycerol kinase coding sequence, showing all known mutations. Mutations and a coding polymorphism described in this study are shown above the schematic. Patient data from a further three studies are summarised below. "atg" marks the first codon, A1 and A2 indicate two of the three polyadenylation signal sequences, and the 3' UTR is shaded.

patient 5 contained a mixture of alternative transcripts with and without the $18 \mathrm{bp}$ insertion (exon $8 \mathrm{~A}$ ) originally described in a fetal brain clone. The two forms occurred in approximately equal amounts. Most of the transcripts also contained the other alternatively spliced exon, exon 18, as the RT-PCR product was of the appropriate size for the larger cDNA, and this was the major sequence ladder observed by direct sequencing.

RT-PCR product representing the 5 ' portion of the GK transcript from patient 4 appeared to be abnormal as it gave three bands (fig 3A). Each PCR product was purified and reamplified with nested primers (GK31 with GK29) before sequencing. The smallest band represented a normal transcript with exons 1 to 7 . The middle band was identical to exon 1 and exon 2, a unique sequence of $212 \mathrm{bp}$ replaced exon 3, and exons 4 to 7 were normal. The largest band matched exons 1 to 7 , with the same unique sequence this time inserted between exons 3 and 4. Comparison with sequences deposited from a Xp22 contig (AC005913) shows that the additional $212 \mathrm{bp}$ are part of the $10 \mathrm{~kb}$ intron 3. Primers designed to flank this $212 \mathrm{bp}$ insertion were used to amplify the intronic sequence and confirm a mutation which creates a new splice site, as well as a polymorphism within the inserted sequence (fig 3B).

Genomic PCR products for individual exons were prepared from patients $1,2,3$, and 6 . A preliminary screen by SSCP was carried out for the first four sets of samples with exons $2,3,4$, $5,6,7,8,11,12,13,14,15,16,17$, and 18 .
This analysis gave reproducible band shifts for patient 1 and patient 2 in exon 14, for patient 3 in exon 11 , and for patient 6 in exon 8 . Sequencing of exon 14 from patients 1 and 2 showed the presence of a premature termination codon Q403X (C1321T) (fig 4A). Similarly, patient 3 was found to have a transversion in exon 11 , which also introduced a stop codon (A1042T, R310X) (fig 4B). No other changes were detected in these patients after sequencing $~ 50 \%$ of the $G K$ gene at the genomic level. The band shift seen in exon 8 for patient 6 arises from a base change in the $3^{\prime}$ flanking intron (IVS8+17 C to A) and probably represents a polymorphism within a region of $59 \mathrm{bp}$ consisting predominantly of poly A (GenBank entry AJ252557). Therefore, the remainder of the gene was systematically sequenced. The only alteration in the coding sequence is silent. This is a transition in the third base of codon 55 (CAG to CAA) in exon 3 (fig 4C), which is heterozygous in the mother of the patient. This latter change removes a restriction site for AvaII (fig 4D). Potentially, such silent base changes can alter exonic splicing elements, but in a screen of $40 \mathrm{X}$ chromosomes, five were found with the CAA codon (not shown). As it is unlikely that such a high frequency of mutation would exist within a normal population, this suggests that the nucleotide change represents a polymorphism.

\section{Discussion}

The C256R change seen in the DNA from patient 5 is likely to represent the true mutation in this family, firstly because it segregates with 
the biochemically affected males, and secondly it alters a highly conserved amino acid. The residue at this position is always cysteine in the human testis specific genes, the $\mathrm{X}$ linked gene of other mammals (rat and mouse), and the putative glycerol kinase genes of $S$ cerivisiae and $C$ elegans. In Mycoplasma genitalium it is valine and in the bacterial glycerol kinase genes it is also non-polar (either I or A). Similarly, the equivalent position in the xylulose kinases for $E$ coli and $H$ influenzae is occupied by a glycine. This amino acid lies close to D259, the residue equivalent to D245 in E coli glycerol kinase, ${ }^{14}{ }^{15}$ which forms part of the glycerol binding pocket at the active site of the bacterial enzyme. The introduction of a charge at this position may be disrupting the three dimensional organisation of the enzyme active site, resulting in a decreased level of enzyme activity.

With patient 4 , no exonic mutations were evident, but the pattern of cDNA species observed following RT-PCR is consistent with a mutation in intron 3 creating an additional splice junction, and this has subsequently been confirmed. The introduction of the novel exon causes premature termination of the gene product by introducing a frameshift into the ORFs of both abnormally sized transcripts (fig 3C). In this case it is unclear whether the disease arises as the result of simple haploinsufficiency or owing to interference by the alternative products. In bacteria, it is known that GK can be present as part of a protein complex and may also form homodimers or homotetramers. ${ }^{14}$ The rat glycerol kinase protein was initially isolated as a dimer and reported as an ATP dependent transporter of activated glucocorticoid receptor complexes. ${ }^{16}$ Currently, the detail of potential sites of interaction within mammalian GK is unknown. In the familial cases 1 and 2 , and patient 3 , the GK gene mutations were shown to be termination codons occurring within the first two thirds of the exonic structure. This may explain the failure of RT-PCR, as other gene transcripts have been found to be subject to a high rate of degradation when similarly mutated..$^{17}$

No evidence for gene mutation was found in the genomic DNA of patient 6 , who was referred as a suspected, rather than a confirmed, case. However, a polymorphism in exon 3 was detected. Additional differences were confined to intronic sequences and are not predicted to be deleterious.

With this study, the total number of independent cases of GKD that have been analysed at the nucleotide level is brought to $11 .{ }^{4519}$ The distribution and the nature of the mutations are illustrated in fig 5. There is no obvious clustering within the gene and no single type of mutation appears to be more common (two splice site, two partial deletions, three terminations, and four missense mutations). All the missense mutations are at highly conserved residues. Patient 5 has been discussed above. The other three residues involved in substitutions, T278, D440, and W503, all occur in blocks of amino acids which show absolute identity between known glycerol kinases, including many from bacteria. Fur- thermore, the equivalent regions of bacterial gluconokinases and xylulokinases are conserved at these residues. In the cases of splice site mutations, deletions, and terminations, all result in premature termination of the protein, with or without novel C-terminal sequences.

As has been stated previously, each family has a unique mutation, with no correlation between the genotype and the observed phenotype. Indeed, the severity of the disease is highly variable within a single family, both between subjects of the same generation and across generations. This has several implications for diagnostic purposes. Firstly, it is not possible to predict the severity of the disease in potential affected family members. This means that prenatal diagnosis is not straightforward in isolated GKD, but assessment of the neonate to determine whether dietary modification is required is important for disease management. Secondly, where possible, the grandparental origin of the mutation is a useful indicator to allow identification of extended family members who are at risk of being obligate carriers. Lastly, isolated GKD within a family usually comes to light when a child has a sufficiently severe phenotype for referral to biochemical and genetics specialists. Among the cases here, and previous reports, many subjects reach adulthood without any acute episodes that would merit such investigations. This may indicate that the actual number of cases of isolated GKD is higher than the published reports suggest. At least some of these patients are being treated for the wrong condition, as the test for raised blood triglyceride also detects raised blood glycerol, but does not distinguish between the two. Conversely, as exemplified by the three brothers in the second generation of the pedigree for case 5, a mixture of hypercholesterolaemia and triglyceridaemia can occur in GK families. Here not all of the raised triglyceride is attributable to the raised blood glycerol. As a result, it is important not to confuse symptoms of a more serious condition with benign adult GKD.

The lack of phenotype/genotype correlation means that there are probably modifiers elsewhere in the genome that contribute to the severity of isolated GKD. These may include polymorphic variants of the constituents of the mitochondrial pathways that regulate the production of ATP, for example, genes for the enzymes in the dihydroxyacetone phosphate shuttle, or the voltage dependent anion channels (VDAC), of which several have been mapped in both mouse ${ }^{20} 21$ and man..$^{22}$ These are proposed to be the site of mitochondrially bound GK and are known to bind the enzyme hexokinase, which competes with GK at the outer mitochondrial membrane. ${ }^{24}$ So far, only one mouse model has been constructed by a knockout of the murine gene. ${ }^{25}$ In this mouse, raised plasma free fatty acids have been implicated in the severe phenotype which shows high mortality within the first three to four days post partum. However, it is unclear whether free fatty acids have a role in modifying the severity of human GKD. Furthermore, it may be necessary to develop a more extensive 
model by selectively introducing mutant chromosomes onto other genetic backgrounds. This would allow the mapping of potential modifying genes, and could include animals carrying the amino acid substitutions observed in reported human cases of GKD.

All sequences are deposited in GenBank under accession numbers AJ252550 to AJ252570. We should like to thank the MRC HGMP Resource centre for the PAC clones isolated during thi study. This work was supported by grants from the MRC and Wellcome Trust.

1 McCabe ER. Disorders of glycerol metabolism. In: Scriver CR, Beaudet AL, Sly WS, Valle D, eds. The metabolic and molecular bases of inherited disease. 7th ed. New York: McGraw- Hill, 1995:1631-43.

2 Rose CI, Haines DSM. Familial hyperglycerolemia. 7 Clin Invest 1978;61:163-70

3 Blomquist HK, Dahl N, Gustafsson L, et al. Glycerol kinase deficiency in two brothers with and without clinical manifestations. Clin Genet 1996;50:375-9.

4 Walker AP, Muscatelli F, Stafford AN, et al. Mutations and phenotype in isolated glycerol kinase deficiency. Am f Hum Genet 1996;58:1205-11.

5 Sjarif DR, Sinke, RJ, Duran M, et al. Clinical heterogeneity and novel mutations in the glycerol kinase gene in three and novel mutations in the glycerol kinase gene in three families with isolated

6 Sargent CA, Affara NA, Bentley E, et al. Cloning of the $\mathrm{X}$-linked glycerol kinase deficiency gene and its identification by sequence comparison to the bacillus subtilis homologue. Hum Mol Genet 1993;2:87-106.

7 Sargent CA, Young C, Marsh S, Ferguson-Smith MA Affara NA. The glycerol kinase gene family: structure of the $\mathrm{Xp}$ gene, and related intronless retroposons. Hum Mol Genet 1994;3:1317-24

8 Walker AP, Muscatelli F, Monaco AP. Isolation of the human glycerol kinase gene by positional cloning. Hum $\mathrm{Mol}$ Genet 1993;2:107-14.

9 Guo W, Worley K, Adams V, et al. Genomic scanning for expressed sequences in Xp21 identifies the glycerol kinase gene. Nat Genet 1993;4:367-72.

10 Bassam BJ, Caetano-Anolles G, Gresshoff PM. Fast and sensitive silver staining of DNA in polyacrylamide gels. sensitive silver staining of DNA in

11 Ioannou PA, de Jong PJ. Construction of bacterial artificial chromosome libraries using the modified P1 (PAC) system. In: Dracopoli et al, eds. Current
New York: John Wiley, 1996.
12 Huq AHMM, Lovell RS, Sampson MJ, et al. Isolation, mapping and functional expression of the mouse $\mathrm{X}$ chromosome glycerol kinase gene. Genomics 1996;36:530-4

13 Kidd A, Sargent C, White I, et al. Family study of isolated glycerol kinase deficiency. $\mathcal{F}$ Med Genet 1998;35:S36.

14 Hurley JH, Faber HR, Worthylake D, et al. Structure of the regulatory complex of Escherichia coli III Glc with glycerol kinase. Science 1993;259:673-7.

15 Pettigrew DW, Smith GB, Thomas KP, Dodds D'NC. Conserved active site aspartates and domain-domain interactions in regulatory properties of the sugar kinase superfamily. Arch Biochem Biophys 1998;349:236-45.

16 Okamoto K, Hirano H, Isohashi F. Molecular cloning of rat iver glucocorticoid-receptor translocation promoter. Biochem Biophys Res Commun 1993;193:848-54.

17 Belgrader P, Maquat LE. Nonsense but not missense mutations can decrease the abundance of nuclear mRNA for the mouse major urinary protein, while both types of mutations can facilitate exon skipping. Mol Coll Biol 1994;14:6326-36.

18 McIntosh I, Hamosh A, Dietz HC. Nonsense mutations and diminished mRNA levels. Nat Genet 1994;4:219.

19 Romero NB, Recan D, Rigal O, et al. A point mutation in the glycerol kinase gene associated with a deletion in the dystrophin gene in a familial X-linked muscular dystrophy: non-contiguous gene syndrome involving Becker muscular dystrophy and glycerol kinase loci. Neuromusc Disord 1997; 7:499-504.

20 Sampson MJ, Lovell RS, Craigen WJ. Isolation, characterization and mapping of two mouse mitochondrial voltagedependent anion channel isoforms. Genomics 1996;33:2838 .

21 Sampson MJ, Lovell RS, Craigen WJ. The murine voltagedependent anion channel gene family. $f$ Biol Chem 1997;272:18966-73.

22 Blachly-Dyson E, Zambronicz E, Yu WH, et al. Cloning and the functional expression in yeast of two human isoforms of the outer mitochondrial membrane channel, the voltage dependent anion channel. 7 Biol Chem 1993;268:1835-41.

23 Blachly-Dyson E, Baldinii A, Litt M, McCabe E, Forte M. Human genes encoding the voltage dependent anion channel (VDAC) of the outer mitochondrial membrane: mapping and identification of two new isoforms. Genomics 1994;20:62-7.

24 Adams V, Griffin L, Towbin J, Gelb B, Worley K, McCabe E. Porin interaction with hexokinase and glycerol kinase: metabolic microcompartmentation at the outer mitochondrial membrane. Biochem Med Metab Biol 1991;45:271-91.

25 Huq AH, Lovell RS, Ou CN, Beaudet AL, Craigen WJ. $\mathrm{X}$-linked glycerol kinase deficiency in the mouse leads to growth retardation, altered fat metabolism, autonomous glucocorticoid secretion and neonatal death. Hum Mol Genet 1997:6:1803-9. 